\title{
C13orf18 and C1orf166 (MULAN) DNA Genes Methylation are Not Associated with Cervical Cancer and Precancerous Lesions of Human Papillomavirus Genotypes in Iranian Women
}

\author{
Amir Sohrabi ${ }^{1}$, Siamak Mirab-Samiee ${ }^{2}$, Marjan Rahnamaye-Farzami ${ }^{3}$, Mitra \\ Rafizadeh $^{4}$, Setareh Akhavan ${ }^{5}$, Mohammad Hashemi-Bahremani ${ }^{4}$, Mohammad \\ Hossein Modarressi ${ }^{6 *}$
}

\begin{abstract}
Background: Nowadays, molecular biomarkers have critical roles for cancer diagnosis and prognosis in clinical laboratories. Human papillomaviruses are the main agents for etiology of cervical carcinoma. The present survey was conducted to evaluate the genes methylation in cervical cancer and precancerous lesions involvement with HPV genotypes. Materials and Methods: C13orf18 and C1orf166 (MUL1 or Mulan) DNA methylation as potential biomarkers and risk factors was investigated in 112 liquid based cytology and Formalin-Fixed ParaffinEmbedded tissue specimens in Iranian females with cervical intraepithelial neoplasia and dysplasia. Results: In this survey, HPV18 $(61.6 \%)$ and HPV16 $(42.9 \%)$ proved to be the most common HPV genotypes identified by In-House Multiplex Real Time PCR. There were no significant relationship between HPV positivity and the methylated DNA genes mentioned above $(p>0.05)$. Conclusions: Our MethyLight data demonstrated that these genes could not be considered as specific, sensitive and suitable prognostic biomarkers in cervical dysplasia related HPV. It is suggested that further studies with more patients should be done on candidate methylated markers in different countries in order to plan for cervical cancer prevention.
\end{abstract}

Keywords: HPV - methylation - C13orf18 - C1orf166 - cervical neoplasia - Iran

Asian Pac J Cancer Prev, 15 (16), 6745-6748

\section{Introduction}

Cervical cancer associated with Human papilomaviruses especially with High Risk (HR) HPV genotypes is one the most public health problems in developing countries such as Iran. Based on the data reported by WHO, the crude incidence and mortality rates of HPV related cervical cancer until 2010 in Iran are about 1.8 and 0.8 per 100,000 per year, respectively. HPVs 16(HR), 18(HR), 31(HR), 6(LR), 11(LR), 33(HR) are the most common HPV genotypes in Iranian women with and without cervical dysplasia (WHO 2010; Laudadido 2013; Shayanfar et al., 2013). It is proposed that individual genetic and epigenetic characteristics accompanied with HPV infection in cervical cancer have important role as main risk factors in cancer progression (Szalmás and Kónya., 2009; Wentzensen et al., 2009; Mohiuddin et al., 2013; Wang ., 2014). Over the last decade, evaluation of methylation biomarkers in cervical precancerous and cancer have been done in various studies. Although wide attempt have been inflected to date, a uniform instructive data for methylation assessment as molecular biomarker has not yet been distinguished for cervical cancer diagnosis and tumor classifying. In recent studies, numerous of methylated genes have been proposed as diagnostic and prognostic biomarkers in cervical carcinoma (Yang et al., 2009; Eijsink et al., 2011; Eijsink et al., 2012; Limpaiboon., 2012; Al-Shabanah et al., 2014; Ma et al., 2014). Hence, we assessed the DNA methylation status of C13orf18 and $\mathrm{C} 1$ orf166 genes as novel potential candidate diagnostic biomarkers in Iranian female population suffering cervical malignancies.

\section{Materials and Methods \\ Clinical specimens \\ From March 2012 to April 2013, a total of 112 samples (48 Liquid Based Cytology and 64 Archival Formalin- Fixed Paraffin-Embedded Tissue (FFPE) specimens) from women with known cervical cancer and precancerous}

${ }^{1}$ Department of Molecular Medicine, School of Advanced Medical Technologies, ${ }^{5}$ Department of Gynecology, Vali-e-asr Hospital, ${ }^{6}$ Department of Medical Genetics, School of Medicine, Tehran University of Medical Sciences, ${ }^{2}$ Food and Drug Laboratory Research Center, Ministry of Health and Medical Education, ${ }^{3}$ Research Center of Health Reference Laboratory, Ministry of Health and Medical Education, Tehran, ${ }^{4}$ Department of Pathology, Imam Hossein Hospital, Shahid Beheshti University of Medical Sciences, Iran *For correspondence: sohrabi@razi.tums.ac.ir,modaresi@tums.ac.ir 
lesions admitted to Tehran Women Central hospital, Imam Khomeini Hospital and Imam Hossein Hospital in Tehran, were collected. All the diagnoses were confirmed by histopathology examinations. A questionnaire was also designed and filled out for each patient and clinical data were entered. The ethical considerations of the study were approved and a consent from was signed by each patient. Then Liquid Based Cytology (LBC) preservatives and FFPE blocks were transported to molecular biology department in health reference laboratory of ministry of health and medical education, Iran. The LBCs were stored at $-20^{\circ} \mathrm{C}$ until experiment.

\section{Extraction of HPV DNA and HPV genotyping}

Viral DNA from LBC preservatives and tissue blocks was extracted using the QIAamp DNA Mini kit and QIAamp DNA FFPE Tissue kit (Qiagen, Germany), according to the manufacturer's instructions. Human Papillomavirus genotyping was performed by In-House Multiplex Real Time PCR developed and designed in our laboratory. The assay has been validated according to determined requirements for assessing the reliability for test (precision, trueness, detection limit, cross reactions and etc) and we were also recognized as a proficient laboratory using this method in 2013 WHO HPV LabNet Proficiency Program that can detect HPV6, 11(Low Risk), HPV 16,18,31,33,35,39,45,51,52,56,58,59,68 (High Risk).

\section{Bisulfite modification and qualitative real time PCR methylation analysis}

Bisulfite treatment was carried out on genomic extracted DNA with Epitect Fast DNA Bisulfite Kit (Qiagen ${ }^{\circledR}$, Germany), according to manufacturer's protocol. HeLa S3 (cervical cancer cell line) and health women pooled plasma are used as positive methylated and un methylated healthy control respectively. Briefly, after bisulfite modifications, Qualitative TaqMan Real Time PCR methylation amplifications were performed in a $20 \mu 1$ reaction volume containing $12.5 \mu 1$ 2X Premix Ex Taq (Probe qPCR) (TaKaRa, Japan), $10 \mathrm{pmol} / \mu 1$ each primers and probes (C13orf18: FP; 5'-TTTTTAGGGAAGTAAAGCGTCG-3', RP; 5'-ACGTAATACTA AACCCGAACGC-3' and TaqMan Probe; Yakima Yellow 5'-AGATGGAAG AAATT TTGGAGAT GC GCGTT-3' BHQ1) (C1 orf166: FP; 5'-TGGCGAGTTAGTTATGGTT AGT G C-3', RV; 5'-TCTCCCCAA CCGAATATCGA-3' and TaqMan Probe; FAM 5'-TTATGGAGCGGTGG

GTGGTCGTTTAG- 3' BHQ1) (TIB MOLBIOL ${ }^{\circledR}$, Germany) (Yang et al., 2009; Eijsink et al., 2011; Eijsink et al., 2012), and 5-6 $\mu 1$ DNA extracted. These reaction mixtures were amplified in a Rotor Gene 6000 (Corbett Life Science), by using the following conditions: $95^{\circ} \mathrm{C}$; $90 \mathrm{~s}$ for initial enzyme activation, for 50 cycles $95^{\circ} \mathrm{C} ; 5 \mathrm{~s}$, and $60^{\circ} \mathrm{C}$; 60s (with fluorescence detection in Green, Yellow Channels).

\section{Statistical analysis}

In order to determine any significant relationship between the DNA methylation of C13orf18 and c1orf166 genes and existence of Human Papillomaviruses, an independent test was used. All tests were performed at the significant level of 0.05 using SPSS software version 18.5.

\section{Results}

Human Papillomviruses were detected in 105 of $112(93.7 \%)$ LBC and Archival FFPE tissue specimens by In-House Multiplex Real Time PCR method. Overall, among the HPV genotypes, HPV18(69/61.6\%), HPV16 (48/42.9\%), HPV58(18/16.1\%), HPV68(16/14.3\%), HPV31(15/13.45), HPV52(15/13.45), HPV59(14/12.5\%), HPV11(13/11.6\%), HPV35(12/10.7\%), HPV56(11/9.8\%), HPV6(9/8\%), HPV39(6/5.4\%), HPV45(4/3.6\%), HPV33(3/2.7\%), HPV51(2/1.8\%) and HPV negative $(7 / 6.3 \%)$ were dominant genotypes in women with cervical dysplasia, respectively. Among samples with the HPV mix genotypes, HPV 16, 18 [13/105 (12.4\%)] were the prevalent combination genotypes. The age range of women was 23-90 years and the mean age (SD) was $42.73( \pm 11.36)$ years old. HPV was detected in $68(64.8 \%)$ of the population study in ages between $31-50$ years old. The frequency rates of DNA Methylation for C13orf18 and C1orf166 genes were $65(58 \%)$ vs $45(40 \%)$, respectively. There were no statistical significance $(\mathrm{p} \geq 0.05)$ between the existence of HPV genotypes infections and C13orf18 and C1orf166 DNA genes 


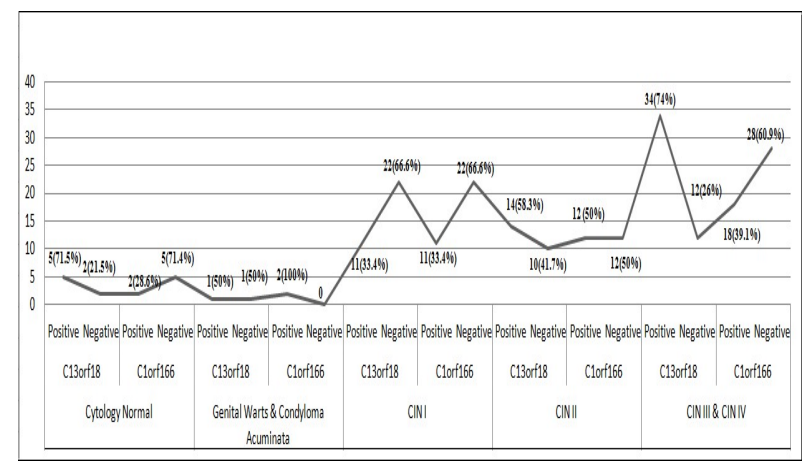

Figure 1. Frequency Rate of C13orf18 and C1orf166 DNA Genes Methylation in Cervical Disorders

methylation in women with cervical disorders. The details of prevalence of HPV genotypes and cervical disorder and Cervical Intraepithelial Neoplasia, and frequency rate of DNA genes methyaltion were summarized in Table 1 and Figure 1.

\section{Discussion}

Cervical carcinoma as a social problem with high morbidity and mortality in the worldwide is discussed. Insomuch, human papillomaviruses genotypes are considered as the main cause of malignancies particulary cervical cancer. Epigenetic and polymorphisms patterns as diagnostic biomarker and HPV genotypes detection can be used for determining appropriate treatment strategy for early stages of cervical cancer and increase survival in cervical malignancies (Nunobiki et al., 2011; Hwang and Shroyer 2012; Wang 2014). Therefore, the extensive studies have been done on the methylation analysis of various genes in different societies (Yang et al., 2009; Eijsink et al., 2011; 2012; Snellenberg et al., 2012; Sun et al., 2012; Banzai et al., 2013; Lan et al., 2013; AlShabanah et al., 2014; Ma et al., 2014). Functional and MethyLight analysis of these genes shown that C13ORF18 gene might represents cervical cancer suppressive activities upon re-expression and C1ORF166, now known as MUL1 or MULAN, is a RING finger E3 ubiquitin ligase that regulates the organelle's dynamics and NF-kB signaling. It is possible that these genes have functional role during cervical tumor development and progression (Li et al., 2008; Yang et al., 2009; Huisman et al., 2013). The distribution of HPV 16 and HPV 18 along with other high risk HPV genotypes in this study is similar to other surveys (Clifford et al., 2005; Lee et al., 2011; Kasamatsu et al., 2012; Manjari and Sweta., 2012; Hamzi Abdul Raub et al., 2013; Turki et al., 2013; Wang et al., 2013; Othman and Othman., 2014). Although the case studies have been conducted on methylation status of C13ORF18 and C1orf166 genes (Yang et al., 2009; Eijsink et al., 2011; Eijsink et al., 2012). Likely, the methylated genes could be considered as proper diagnostic biomarkers in cervical cancer mid HPVs (Dueñas-González et al., 2005; Szalmás et al.,2009; Wentzensen et al., 2009; Nogueira da Costa and Herceg., 2012). However, they were detected of DNA methylated genes for C13orf18; 65 (58\%) and C1orf166; 45 (40\%) in this research, but we did not found any statistical significances between women with cervical dysplasia and HPV genotypes previously found positive $(p>0.05)$. Therefore, introducing these methylated genes as prognostic biomarkers of cervical cancer needs further studies with genetic patterns variation and various designs and acceptable number of clinical specimens.

In Conclusions, Obviously increased cervical cancer and precancerous lesions with HPV genotypes infection were seen in different regions of world, hence, further investigations should be emphasized for detection and development of molecular diagnostic biomarkers in cancers. More investigations on $\mathrm{CpG}$ methylation regions in different countries with more clinical samples can be helpful in proper selection of specific prognostic biomarkers and treatment procedures in cancers.

\section{Acknowledgements}

We are grateful and indebted to laboratories staff and nurses of Tehran Women Center Hospital, Imam Khomeini Hospital and Imam Hossein Hospital especially Mrs. Amirani, Mrs.Asadian \& Mrs. Moshtagh for helping us in gathering of specimens.

\section{References}

Al-Shabanah OA, Hafez MM, Hassan ZK, et al (2014). Methylation of SFRPs and APC Genes in ovarian cancer infected with high risk human papillomavirus. Asian Pac J Cancer Prev, 15, 2719-25.

Banzai C, Nishino K, Quan J, et al (2013). Promoter methylation of DAPK1, FHIT, MGMT, and CDKN2A genes in cervical carcinoma. Int J Clin Oncol, 19, 127-32.

Clifford GM, Rana RK, Franceschi S, et al (2005). Human papillomavirus genotype distribution in low-grade cervical lesions: comparison by geographic region and with cervical cancer. Cancer Epidemiol Biomarkers Prev, 14, 1157-64.

Dueñas-González A, Lizano M, Candelaria M, et al (2005). Epigenetics of cervical cancer. An overview and therapeutic perspectives. Mol Cancer, 25, 4, 38.

Eijsink JJH, Lendvai A, Deregowski V, et al (2012). Four-gene methylation marker panel as triage test in highrisk human papillomavirus positive patients. Int J Cancer, 130, 1861-9.

Eijsink JJ, Yang N, Lendvai A, et al (2011). Detection of cervical neoplasia by DNA methylation analysis in cervico-vaginal lavages, a feasibility study. Gynecol Oncol, 120, 280-3.

Hamzi Abdul Raub S, Isa NM, Zailani HA, et al (2013). Distribution of HPV genotypes in cervical cancer in multiethnic Malaysia. Asian Pac J Cancer Prev, 15, 651-6.

Huisman C, Wisman GB, Kazemier HG, et al (2013). Functional validation of putative tumor suppressor gene C13ORF18 in cervical cancer by artificial transcription factors. $\mathrm{Mol}$ Oncol, 7, 669-79.

Hwang SJ, Shroyer KR (2012). Biomarkers of cervical dysplasia and carcinoma. J Oncol, 507286, doi: 10.1155/2012/507286.

Kasamatsu E, Cubilla AL, Alemany L, et al (2012). Type-specific human papillomavirus distribution in invasive cervical carcinomas in paraguay, a study of 432 cases. J Med Virol, 84, 1628-35.

Lan VTT, Thuan TB, Thu DM, et al (2013). Methylation profile of BRCA1, RASSF1A and ER in Vietnamese women with ovarian cancer. Asian Pac J Cancer Prev, 14, 7713-8.

Laudadido J (2013). Human papillomavirus detection: testing methodologies and their clinical utility in cervical cancer screening. Adv Anat Pathol, 20, 158-67.

Lee JH, Lee NW, Hong SW, et al (2011). Establishment of 
an efficient multiplex real-time PCR assay for human papillomavirus genotyping in cervical cytology specimens: comparison with hybrid capture II. Cytopathology, 22, 261-8.

Li W, Bengtson MH, Ulbrich A, et al (2008). Genome-wide and functional annotation of human E3 ubiquitin ligases identifies MULAN, a mitochondrial E3 that regulates the organelle's dynamics and signaling. PLoS One, 3, 1487.

Limpaiboon T (2012). Epigenetic aberrations in cholangiocarcinoma: potential biomarkers and promising target for novel therapeutic strategies. Asian Pacific J Cancer Prev, 13, 41-5.

Ma JQ, Kurban S, Zhao JD, et al (2014). Epigenetic regulation of human riboflavin transporter 2(hRFT2) in cervical cancers from uighur women. Asian Pac J Cancer Prev, 15, 2485-9.

Manjari M, Sweta S (2012). Detection of HPV by PCR-a novel step in the prevention of cancer cervix. J Obstet Gynaecol India, 62, 188-91.

Mohiuddin MK, Chava S, Upendrum P, et al (2013). Role of human papilloma virus infection and altered methylation of specific genes in esophageal cancer. Asian Pac J Cancer Prev, 14, 4187-93.

Nogueira da Costa A, Herceg Z (2012). Detection of cancerspecific epigenomic changes in biofluids: powerful tools in biomarker discovery and application. Mol Oncol, 6, 704-15.

Nunobiki O, Ueda M, Toji E, et al (2011). Genetic polymorphism of cancer susceptibility genes and HPV infection in cervical carcinogenesis. Patholog Res Int, 364069, doi: $10.4061 / 2011 / 364069$.

Othman N, Othman NH (2014). Detection of human papillomavirus DNA in routine cervical scraping samples: use for a national cervical cancer screening program in a developing nation. Asian Pac J Cancer Prev, 15, 2245-9.

Snellenberg S, De Strooper LM, Hesselink AT, et al (2012). Development of a multiplex methylation-specific PCR as candidate triage test for women with an HPV-positive cervical scrape. BMC Cancer, 23, 12:551.

Shayanfar N, Hosseini N, Panahi M, et al (2013). Detection of mucosal type human papillomavirus in cutaneous squamous cell carcinoma in Iran. Pathol Res Pract, 209, 90-4.

Sun LL, Cao DY, Yang JX, et al (2012). Population-based casecontrol study on DAPK1, RAR-2 and MGMT methylation in liquid-based cytology. Arch Gynecol Obstet, 285, 1433-9.

Szalmás A, Kónya J (2009). Epigenetic alterations in cervical carcinogenesis. Semin Cancer Biol, 19, 144-52.

Turki R, Sait K, Anfinan N, et al (2013). Prevalence of human papillomavirus in women from Saudi Arabia. Asian Pac J Cancer Prev, 14, 3177-81.

Yang N, Eijsink JJH, Lendvai A, et al (2009). Methylation markers for CCNA1 and C13ORF18 are strongly associated with high-grade cervical intraepithelial neoplasia and cervical cancer in cervical scrapings. Cancer Epidemiol Biomarkers Prev, 18, 3000-7.

Wang YY, Li L, Wei S, et al (2013). Human papillomavirus (HPV) infection in women participating in cervical cancer screening from 2006 to 2010 in Shenzhen city, south China. Asian Pac J Cancer Prev, 14, 7483-7.

Wang ZM (2014). PAX1 methylation analysis by MS-HRM is useful in triage of high-grade squamous intraepithelial lesions. Asian Pac J Cancer Prev, 15, 891-4.

Wentzensen N, Sherman ME, Schiffman M, et al (2009). Utility of methylation markers in cervical cancer early detection: Appraisal of the state-of-the-science. Gynecol Oncol, 112, 293-9.

WHO/ICO HPV Information Centre (2010). Human papillomavirus and related cancers, specific Iran summary report. Available at http://www.who.int/hpvcentre. 